\title{
The last word(s) on CPOD 2013
}

\section{Frithjof Karsch*}

Physics Department, Brookhaven National Laboratory, Upton, NY 11973, USA

Fakultät für Physik, Universität Bielefeld, D-33615 Bielefeld, Germany

E-mail: karsch@bnl.gov

Higher order moments of net conserved charge fluctuations, in particular net baryon number and net electric charge, are sensitive thermodynamic observables that respond strongly to critical behavior in strong interaction matter. In order to use them also as a sensible probe to detect critical behavior in heavy ion experiments we not only need to better understand the relation between chemical freeze-out in heavy ion collision and the QCD phase boundary, we also need to verify that charge fluctuations measured experimentally indeed correspond to thermal conditions as described by equilibrium QCD. This requires a model independent characterization of thermal conditions for which cumulants of conserved charge fluctuations themselves are ideally suited.

8th International Workshop on Critical Point and Onset of Deconfinement,

March 11 to 15, 2013

Napa, California, USA

\footnotetext{
*This work has been supported in part by contract DE-AC02-98CH10886 with the U.S. Department of Energy.
} 


\section{Introduction}

A major incentive for the heavy ion research programs at RHIC, the SPS and LHC as well as for the planning of the future facilities FAIR and NICA is the prospect to gain insight into the supposably rich phase structure of strongly interacting matter at high temperature and non-zero net baryon number density. Beam energy scan (BES) programs at RHIC and the SPS are performed to search for evidence for a second order phase transition point, the critical endpoint, which has been postulated to exist at high temperature and non-zero net baryon number density or, equivalently, non-zero baryon chemical potential $\left(\mu_{B}\right)[1]$.

Experiments at RHIC and LHC provide plenty of evidence that locally equilibrated hot and dense matter, the quark-gluon plasma, is formed in ultra-relativistic heavy ion collisions. Thermal photons emitted from the early phase of the expanding fireball [2] suggest that the temperature was at least twice as large as the QCD transition temperature, $T_{c} \simeq 155 \mathrm{MeV}[3,4]$. The analysis of elliptic flow of different particle species provides evidence that the observed collective behavior arises from the interaction of partonic degrees of freedom [5]. Their dominance, however, gets lost at low beam energies. The BES at the SPS provided first evidence for this so-called ${ }^{1}$ 'Onset of Deconfinement' [6], i.e., it seems that beam energies larger than $\sqrt{s_{N N}} \simeq(5-7) \mathrm{GeV}$ are needed to create in heavy ion collisions sufficiently dense matter so that the phase boundary to a high density deconfined state of matter can possibly be crossed. Indications for the onset of deconfinement now are also found in data for the elliptic flow parameter $v_{2}$ measured by STAR in the BES at RHIC [7]; for $\sqrt{s_{N N}} \leq 11.5 \mathrm{GeV} v_{2}$ for different hadron species no longer scales with the number of constituent quarks. At lower beam energies the flow pattern seems to be controlled by purely hadronic degrees of freedom.

While we gained confidence that a new form of matter has been formed in heavy ion collisions, we are less confident about experimental evidence for the location of the phase boundary that separates the low and high temperature regime and so far we also have no indications for the existence of a critical endpoint. Lattice QCD calculations are ambiguous about the possible existence of a critical point $[8,9,10]$ and first results from the BES at RHIC are far from being conclusive $[11,12,13]$. The analysis of net conserved charge fluctuations of hadrons may improve this situation. They carry information about critical behavior related to the occurrence of a phase transition in the limit of vanishing quark mass, $m_{q}$, as well as to the existence of a critical endpoint at $\mu_{B}>0$ and $m_{q}>0$. However, in order for them to be a sensitive tool for detecting critical behavior in heavy ion experiments it clearly is necessary that the measurable fluctuations are generated close to the QCD phase boundary (for an illustration see Fig. 1 (left)).

It generally is assumed that the fluctuations of conserved charges, measured at RHIC or LHC, are generated at the time of chemical freeze-out. Furthermore, it has been argued that the hadronization temperature, which one may identify with the QCD transition temperature, and the chemical freeze-out temperature, at which the abundances of different particle species observed experimentally is fixed, differ only little [14]. This finds support through the recently performed reanalysis of particle yields at chemical freeze-out and the reconstructed yields at the time of hadronization [15]. This analysis also shows that in particular the yields of protons and anti-protons, which are impor-

\footnotetext{
${ }^{1}$ The start for a systematic search for the Onset of Deconfinement and the location of a Critical Point in the QCD phase diagram gave name to the series of CPOD conferences, which started 2004 with a workshop at the ECT* in Trento.
} 

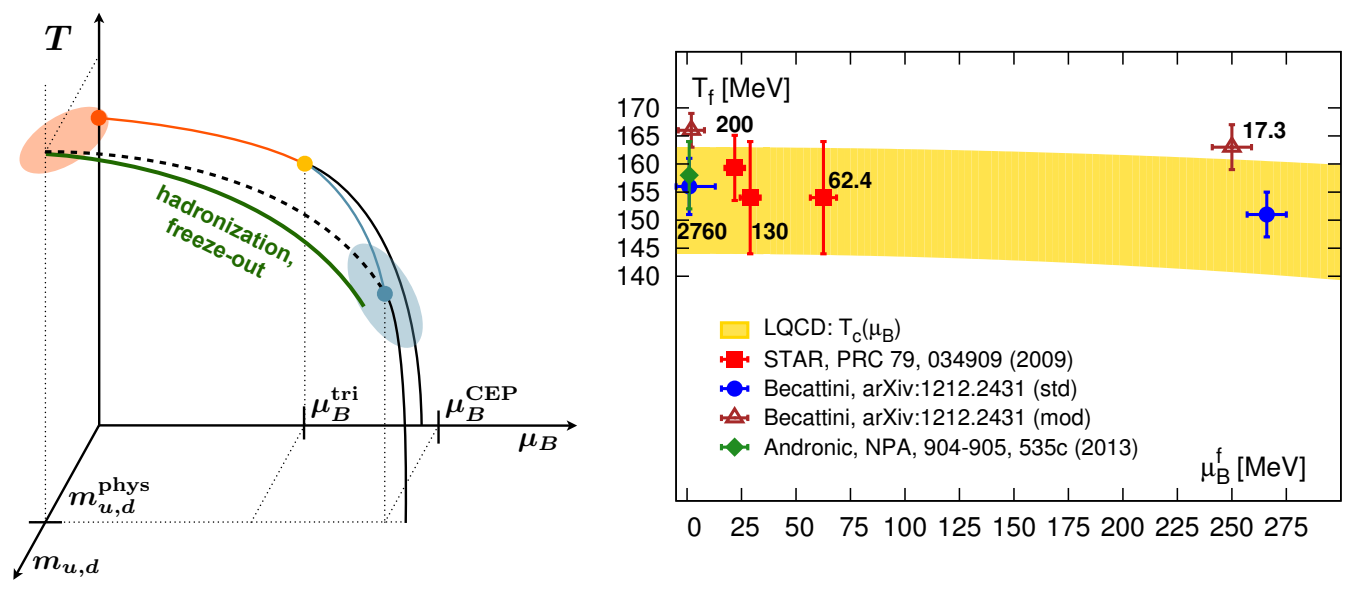

Figure 1: Left: A schematic phase diagram of QCD in the $T-\mu_{B}$ and light quark mass space. It shows the phase transition line at vanishing chemical potential as well as a line of second order transitions, that will exist, if a tri-critical point exists in the chiral limit at non-zero baryon chemical potential. The dotted line shows the crossover transition line of QCD at physical values of the quark mass that ends in a critical point. Also indicated is the freeze-out or hadronization line determined experimentally. Shaded areas indicate the critical region in the vicinity of the critical endpoint and the chiral transition at $\mu_{B}=0$, respectively. Right: The QCD transition temperature as function of the baryon chemical determined from the maximum of the chiral susceptibility [4] and the scaling behavior of the second derivative of the chiral condensate with respect to the chemical potential [18]. Data points show chemical freeze-out temperatures determined from particle yields measured at the LHC [15, 16], the SPS [15] and in the BES at RHIC [17]. The difference between triangles and circles reflects the influence of proton anti-proton annihilation processes, that take place after hadronization, on the determination of the freeze-out parameters [15].

tant for the generation of net baryon number fluctuations, may be distorted due to non-equilibrium, annihilation processes occurring between the time of hadronization and the freeze-out of the bulk of particle species. This may also have consequences for the interpretation of experimentally observed charge fluctuations.

In order to use fluctuation observables in the search for critical behavior it clearly is mandatory to understand at which time these fluctuations are generated. I.e. are the fluctuations of thermal origin and, if so, what are the thermal conditions probed by these observables? Do they correspond to a well defined point in the QCD phase diagram? If so, how is this point related to the QCD transition line? Answering these question also requires that we are clear about our notion of the QCD transition temperature as well as the freeze-out temperature. We will address some of these questions, which have intensively been discussed at CPOD 2013, in the following.

\section{Pseudo-critical temperatures and freeze-out temperatures}

Searches for critical behavior in the BES at RHIC and the SPS to a large extent rely on the expectation that changes in correlation lengths that will arise in the vicinity of a critical point give rise to non-monotonic structures in observables that are sensitive to fluctuations generated at the time of hadronization or chemical freeze-out. This assumes that the line of chemical freeze-out parameters $\left(T_{f}, \mu_{B}^{f}\right)$ is well defined and closely follows the QCD transition line, which eventually may end in a 
true second order phase transition point. Of course, freeze-out itself is not an exact notion. Not all particles will hadronize at the same time, some particle species may fall out of equilibrium earlier than others and the decay of resonances at later times requires feed-down corrections.

Also the notion of a transition temperature in QCD is not exact. A true phase transition with a unique critical temperature only exists in the chiral limit, $m_{q} \rightarrow 0$. For $m_{q}>0$ one has several options to define pseudo-critical temperatures. However, also other indicators for the transition are used, that have no direct or only a weak link to critical behavior. It thus is important to be clear about the notion of a pseudo-critical temperature and the way it is related to critical behavior in QCD as well as the chemical freeze-out temperature.

Pseudo-critical temperatures. In the absence of a true phase transition pseudo-critical temperatures are introduced to characterize the crossover from the low temperature, chiral symmetry broken to the high temperature, chirally symmetric phase of QCD. Pseudo-critical temperatures reflect properties of the non-analytic part of the free energy density. In the limit of vanishing explicit symmetry breaking, this non-analyticity shows up as a divergence in a sufficiently high order derivative of the free energy density. In that limit all pseudo-critical temperatures approach the unique critical temperature. In order not to be overwhelmed by regular contributions to the free energy density one clearly should take care that the observables used to define a pseudo-critical temperature strongly couple to the singular part and, preferably, diverge at the critical point, i.e., in the limit of vanishing explicit symmetry breaking.

In QCD chiral symmetry is explicitly broken by the non-vanishing light quark masses. The location of a peak in the disconnected part of the chiral susceptibility, $\chi_{\text {disc }} \sim \partial^{2} \ln Z / \partial m_{q}^{2}$, provides a well defined pseudo-critical temperature. The peak hight of $\chi_{\text {disc }}$ will increase with decreasing light quark mass $m_{q}$ and will lead to a divergent susceptibility in the chiral limit. Its location defines the chiral phase transition temperature.

In Fig. 1 (right) we show results for the pseudo-critical temperature $T_{c}\left(\mu_{B}\right)$, determined from the position of a peak in $\chi_{\text {disc }}$ at $\mu_{B}=0$ [4], and its leading order correction in $\mu_{B} / T$ [18],

$$
T_{c}\left(\mu_{B}\right)=\left(154(9)-\kappa_{B}\left(\frac{\mu_{B}}{T}\right)^{2}\right) \mathrm{MeV} \text { with } \kappa_{B}=0.0066(7) .
$$

This is consistent with a determination of $T_{c}\left(\mu_{B}\right)$ that also is based on an analysis of the chiral susceptibility $[3,19]$.

Strange quark number susceptibility and the Polyakov loop. In the chiral limit the QCD phase transition is expected to belong to the universality class of three dimensional $O(4)$ symmetric spin models ${ }^{2}$. Derivatives of the order parameter with respect to $T$ or $m_{q}$ become singular in that limit. Divergences in energy like observables however only arise in third derivatives of the partition function with respect to temperature [20]. I.e., second derivatives with respect to $T, \mu_{B}^{2}$ or $\mu_{S}^{2}$ stay finite even in the chiral limit. Quadratic fluctuations of conserved charges, which are only first derivatives of the QCD partition functions with respect to $\mu_{B}^{2}$ or $\mu_{S}^{2}$, show even less sensitivity for the singular structure of the QCD partition function and also their slope as function of temperature will stay finite in the chiral limit. In fact, extracting information on critical behavior from quadratic quark number fluctuations would require to become sensitive to a sub-leading temperature dependence, $\sim\left(T-T_{C}\right)^{1+|\alpha|}$. Extracting a transition temperature from them thus is as difficult

\footnotetext{
${ }^{2}$ This is not undisputed as the influence of the axial anomaly on the chiral phase transition still is not well controlled.
} 
as extracting the transition temperature directly from the energy density of QCD. They are thus not well suited for the definition of a pseudo-critical temperature. Similar difficulties arise for the Polyakov loop expectation value $(P)$ for which a direct relation to derivatives of the QCD partition function does not exist, although $P$ is likely to couple to energy like observables.

Nonetheless, the strange quark number susceptibility and the Polyakov loop expectation value have been used to determine crossover temperatures. These observables lead to estimates for the crossover temperature, which are about $15 \mathrm{MeV}$ larger than the value extracted from the chiral susceptibility [3]. This discrepancy may persist even in the chiral limit as the singular contributions to these observables may be strongly suppressed and overwhelmed by contributions from the regular part of the QCD partition function. The difference between these crossover temperatures and the pseudo-critical temperature for the chiral transition led to speculations about a possible deconfinement transition being distinct from the chiral transition. We will discuss this in section 3 .

Freeze-out temperatures. The line of freeze-out parameters is commonly determined by comparing experimentally measured yields for various particle species with a statistical model calculation, i.e. the hadron resonance gas (HRG) model [21]. This does provide a quite satisfactory characterization of thermal conditions at the time of freeze-out at the SPS [15] and in the BES at RHIC [17]. However, measurements of particle yields at the LHC made it obvious that this approach needs to be refined in order to deal with the observed proton and anti-proton yields[15, 16]. As pointed out in Ref. [15] the incorporation of non-equilibrium processes arising in particular from proton anti-proton annihilation and regeneration processes may improve thermal fits, but will lead to somewhat larger freeze-out temperatures [15] also at lower beam energies. This temperature may even agree with the hadronization temperature and thus should be close to the QCD transition temperature. Incorporating these effects in the determination of freeze-out parameters, however, requires the reconstruction of the latest state of hadronic chemical equilibrium using a transport model like UrQMD. This introduces additional model dependences in the determination of freezeout parameters.

As shown by the triangles and circles in Fig. 1 (right) the standard as well as the modified approach to the determination of freeze-out parameters lead to temperatures consistent with the QCD transition temperature. Although this is gratifying, it also leaves us with a conceptual problem as it seems that freeze-out happens indeed close to the QCD phase boundary, one may wonder whether an uncorrelated HRG still captures correctly the strong interactions that take place close to the QCD phase boundary. At least one should verify that in this regime the HRG model still is a good approximation to QCD thermodynamics and, in particular, provides an appropriate characterization of the chemical composition of strongly interacting matter in the crossover region of the QCD transition.

\section{The chiral transition and deconfinement}

Recent analyzes of quadratic charge fluctuations $[22,23]$ have shown that these are indeed well described by the HRG for $T \lesssim 160 \mathrm{MeV}$. Moreover, strangeness fluctuations seem to be in agreement with HRG model calculations even at $T \simeq 170 \mathrm{MeV}$. This led to speculations that strangeness may deconfine only well above the chiral transition [24]. We will discuss here that higher order cumulants do not provide any hints for such an assertion. 
Chiral transition. Cumulants of fluctuations of net conserved charges of QCD, i.e., net baryon number, electric charge and strangeness, are obtained from derivatives of the QCD partition function with respect to the corresponding charge chemical potentials,

$$
\chi_{i j k}^{B Q S}\left(\mu_{B}, \mu_{Q}, \mu_{S}\right)=\frac{1}{V T^{3}} \frac{\partial^{i+j+k} \ln Z\left(V, T, \mu_{B}, \mu_{Q}, \mu_{S}\right)}{\partial\left(\mu_{B} / T\right)^{i} \partial\left(\mu_{Q} / T\right)^{j} \partial\left(\mu_{S} / T\right)^{k}} .
$$

Divergences arising from derivatives of the singular part of the QCD partition function will show up prominently in higher order cumulants $\chi_{i j k}^{B Q S}$. These observables are thus sensitive to the occurrence of the chiral phase transition in QCD. However, at vanishing $\mu_{B}$ only sixth order cumulants will diverge in the chiral limit and will provide an unambiguous signal for the chiral phase transition. For $\mu_{B}>0$ already third order cumulants $(i+j+k=6)$ will diverge. However, the leading divergence is proportional to $\left(\kappa_{B} \mu_{B} / T\right)^{3}$, with $\kappa_{B}$ from Eq. 2.1. For $m_{q}>0$ the amplitude of the divergent term thus is parametrically suppressed and may become important only at large $\mu_{B}$. In any case, an analysis of higher order cumulants of net charge fluctuations is needed in order to use charge fluctuations as a tool to study the QCD chiral transition.

Deconfinement. The inflection point in the quadratic strange quark number susceptibility, $\chi_{2}^{S} \equiv \chi_{002}^{B Q S}$, has been used to define a crossover temperature for the QCD transition [3]. As this temperature is about $15 \mathrm{MeV}$ larger than the pseudo-critical temperature extracted from the peak of the chiral susceptibility and as it agrees with the temperature extracted from the slope of the Polyakov loop, it has been speculated that deconfinement, in particular deconfinement of strange quarks may happen in the chirally symmetric phase [24]. If correct, this would also alter considerably our picture of strange charge fluctuations and their relation to chemical freeze-out.

The analysis of fourth order cumulants of strangeness fluctuations provides further insight into this question [25]. In Fig. 2 (left) we show the difference between the correlation of net strangeness fluctuations with first and third order net baryon number fluctuations, respectively, i.e. $\chi_{31}^{B S}-\chi_{11}^{B S}$. In an uncorrelated gas of hadrons, i.e. the HRG, this difference vanishes. As can be seen from Fig. 2 (left) this is approximately realized only up to $T \simeq 160 \mathrm{MeV}$. The same conclusion can be drawn from the difference of second and fourth order net baryon number cumulants $\chi_{2}^{B}-\chi_{4}^{B}$. In a HRG also this quantity, which in addition includes information on baryons formed from light quarks only, vanishes. Fig. 2 (left) strongly suggests that the strange and non-strange baryon sector behave similar and that both differ significantly from an uncorrelated HRG for $T>160 \mathrm{MeV}$.

Concurrent information on strangeness is also contained in the ratio of mixed susceptibilities $\chi_{211}^{B Q S}$ and $\chi_{121}^{B Q S}$ shown in Fig. 2 (right). At high temperature each of these susceptibilities singles out the same contribution to a free strange quark gas. Their ratio thus will approach unity in the infinite temperature limit. In a gas of uncorrelated hadrons these susceptibilities project onto strange charged baryons, but they give different weight to the charge one and two sectors. The latter is enhanced in $\chi_{121}^{B Q S}$. One thus expects the ratio $\chi_{211}^{B Q S} / \chi_{121}^{B Q S}$ to be less than unity in an HRG. As can be seen this is indeed the case and the ratio is consistent with a HRG model calculation only for $T \lesssim 160 \mathrm{MeV}$.

Also the analysis of other fourth order cumulants [26] leads to the conclusion that the straightforward picture of an uncorrelated HRG breaks down quite abruptly at $T \simeq 160 \mathrm{MeV}$, but provides a rather good description of hadron thermodynamics and chemistry below this temperature. Also in the strange quark sector deviations from the HRG model rapidly become large for $T \geq 160 \mathrm{MeV}$. 

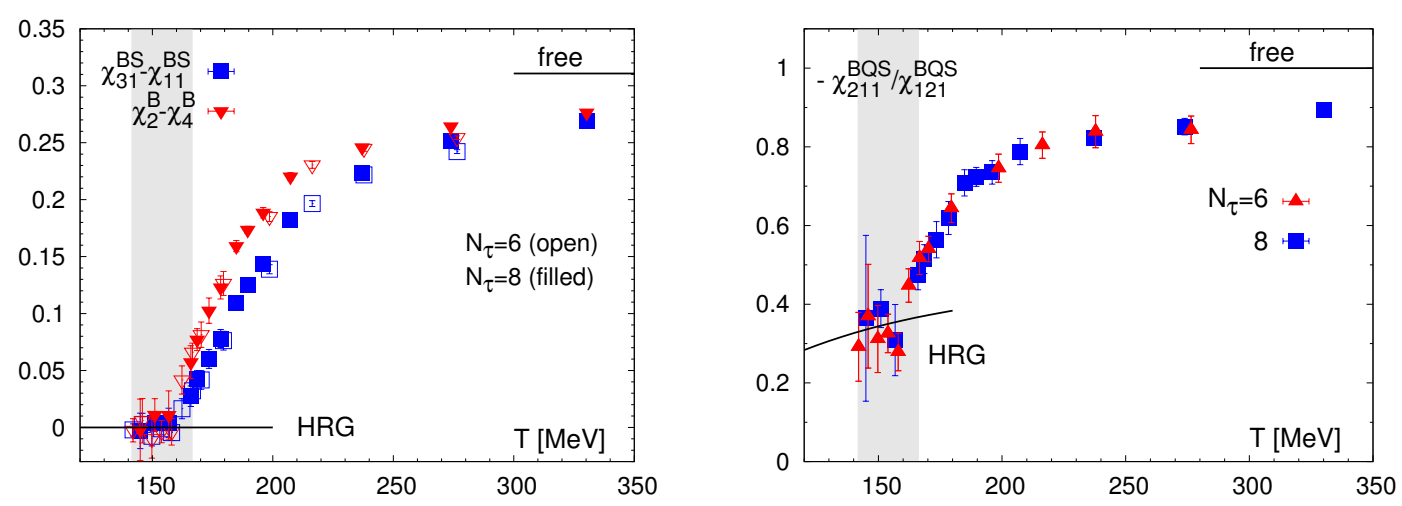

Figure 2: Left: The difference of $4^{\text {th }}$ and $2^{\text {nd }}$ order cumulants combined in such a way that they vanish in an uncorrelated hadron resonance gas (in Boltzmann approximation) [25]. Right: The ratio of two mixed cumulants that project onto the quantum numbers of strange quarks and yield identical results in a free strange quark gas.

Strange hadrons thus are either dissolved or strongly modified above $T=160 \mathrm{MeV}$. This reconfirms that the pseudo-critical temperature extracted from the chiral susceptibility is a good indicator for critical behavior in QCD that also reflects deconfining features of the QCD transition. At present there are no indications that the liberation of light or strange quark degrees of freedom is delayed and would happen at higher temperatures than the chiral crossover.

\section{Freeze-out and higher order cumulants of net charge fluctuations}

Higher order cumulants of net charge fluctuations become increasingly sensitive to critical behavior. However, they 'only' influence the exponentially small tails of charge distributions. This makes it difficult to calculated them with high accuracy in lattice QCD as well as to measure them in heavy ion experiments. Nonetheless, they are appealing because they allow to determine fundamental features of the QCD transition directly by comparing experimental data with a QCD calculation. E.g. one may examine ratios of cumulants that are related to mean $(M)$, variance $\left(\sigma^{2}\right)$, skewness $(S)$ or kurtosis $(\kappa)$ of net charge distributions,

$$
\frac{M_{X}}{\sigma_{X}^{2}}=\frac{\chi_{1}^{X}}{\chi_{2}^{X}}, \frac{S_{X} \sigma_{X}^{3}}{M_{X}}=\frac{\chi_{3}^{X}}{\chi_{1}^{X}}, \kappa_{X} \sigma_{X}^{2}=\frac{\chi_{4}^{X}}{\chi_{2}^{X}}, X=B, Q, S .
$$

The experimental determination of ratios of cumulants of net charge fluctuations itself is not a straightforward measurement. One needs to control the influence of experimental cuts and efficiency corrections. At lower beam energies one may worry whether the exact conservation of charges in a small volume needs to be taken into account and one may need to correct for finite volume effects and volume fluctuations [27].

In order to establish the measurement of fluctuations as a credible tool for the analysis of critical behavior in QCD a first step clearly should be to verify that different cumulant ratios carry information on thermal behavior that corresponds to a unique point in the QCD phase diagram. It thus is important to establish that thermal parameters $\left(T, \mu_{B}\right)$ and eventually also $\left(\mu_{S}, \mu_{Q}\right)$ can be extracted from cumulant ratios without making reference to model calculations. I.e. for consistency 


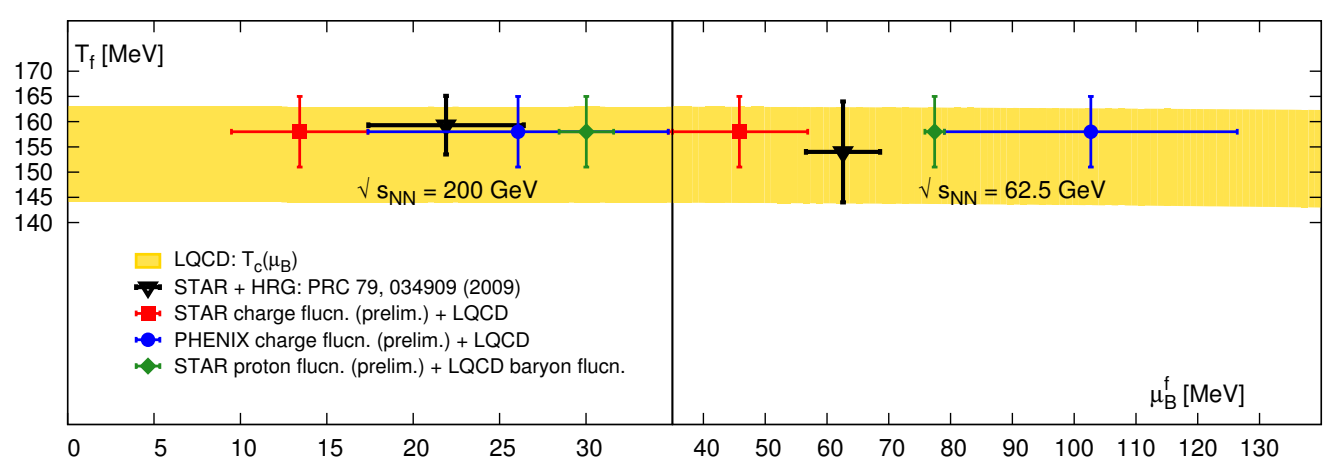

Figure 3: Freeze-out parameters determined from particle yields (triangle) [17] and from net electric charge fluctuations (circles, squares) using the cumulants $\chi_{1}^{Q} / \chi_{2}^{Q}$ and $\chi_{3}^{Q} / \chi_{1}^{Q}$ to fix $\mu_{B}^{f}$ and $T_{f}$, respectively [28, 29]. Also shown is a point obtained by comparing STAR data on net proton number fluctuations (diamond) with lattice QCD calculations for net baryon number fluctuations [28].

these parameters need to be determined by comparing QCD calculations with experiment. E.g. one may extract $\left(T_{f}, \mu_{B}^{f}\right)$ from any independent set of two ratios of cumulants. A convenient way to do so is to use the ratios $\chi_{1}^{X} / \chi_{2}^{X}$ and $\chi_{3}^{X} / \chi_{1}^{X}$ [29]. Other ratios, involving also higher order cumulants, should then lead to a consistent determination of these freeze-out parameters. In the BES at RHIC STAR and PHENIX have measured up to fourth order cumulants of net electric charge fluctuations $[12,13]$ and STAR also presented results on net proton number fluctuations [11]. At present most of these data are preliminary. We use them here to test whether a determination of $\left(T_{f}, \mu_{B}^{f}\right)$ from cumulant ratios as discussed above is feasible at all ${ }^{3}$. Results of such an analysis are shown in Fig. 3 [28]. This necessarily preliminar analysis shows that freeze-out temperature can be extracted from cumulant ratios and are in reasonable agreement with the determination based on hadron yields. The current determination of freeze-out chemical potentials, however, also shows discrepancies that become more obvious at lower beam energies. The discrepancies between the preliminary data on electric charge fluctuations obtained by STAR and PHENIX clearly show that better control over the influence of cuts and efficiency corrections is needed before a reliable determination of freeze-out parameters from cumulant ratios can be performed. At present the analysis that led to Fig. 3 should thus merely be taken as a proof of principle.

\section{QCD critical endpoint}

At least for small values of $\mu_{B}$, or for beam energies $\sqrt{s_{N N}} \gtrsim 20 \mathrm{GeV}$, it seems that the measured net charge fluctuations are generated close to the QCD transition. If this remains to be the case at larger values of the chemical potential one may indeed hope that fluctuations, generated at the time of hadronization, carry information about a nearby critical point and thus allow to detect its location in the QCD phase diagram. The signature for the critical point encoded in the beam energy dependence of e.g. $\left(\kappa \sigma^{2}\right)_{B}$ or $\left(\kappa \sigma^{2}\right)_{Q}$ nonetheless will depend strongly on the relative location of the freeze-out points and the critical point. This is easily illustrated in a model calculation.

\footnotetext{
${ }^{3}$ The measured net proton cumulants may be considered as a proxy for net baryon number fluctuations that can be calculated in lattice QCD. Of course, to some extent this assumes already that an uncorrelated gas of hadrons provides a good description of the thermodynamics at the time of freeze-out.
} 

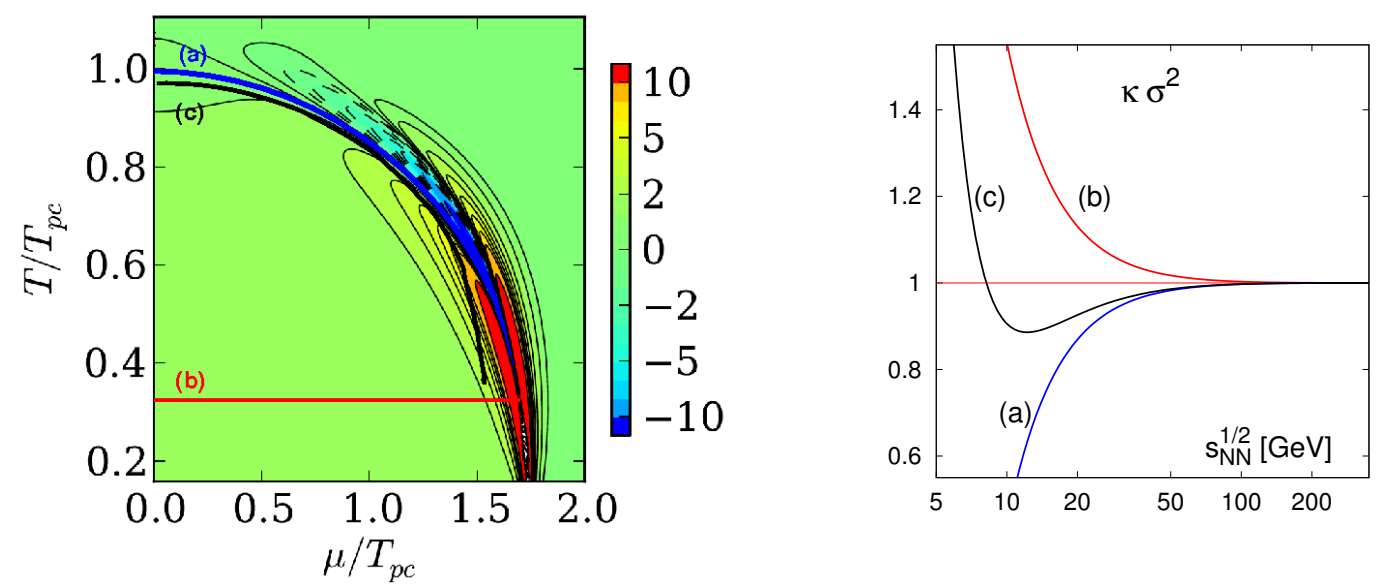

Figure 4: Contour plot for the baryon number kurtosis times variance calculated in the Polyakov-QuarkMeson model [30] (left) and a sketch for the beam energy dependence of this quantity for three different paths in the $T-\mu_{B}$ plane.

In Fig. 4 (left) we show a contour plot for net baryon number kurtosis times variance, $\left(\kappa \sigma^{2}\right)_{B}$, calculated in a mean-field approximation of the quark meson model [30]. The basic features seen in this figure arise from the global $O(4)$ symmetry that the model has in common with QCD and the existence of a critical point in this model. When increasing the baryon chemical potential along the crossover line (path (a)) $\left(\kappa \sigma^{2}\right)_{B}$, decreases, becomes negative and eventually will diverge to minus infinity at the critical point. On the other hand, $\left(\kappa \sigma^{2}\right)_{B}$ stays positive when approaching the critical point at a lower temperature, in particular for $T=T_{C P}$ (path (b)). It will increase with increasing $\mu_{B}$ and eventually will diverge to plus infinity when approaching the critical point ${ }^{4}$. Along the hypothetical freeze-out line (path (c)) $\left(\kappa \sigma^{2}\right)_{B}$ may decrease with increasing $\mu_{B}$, eventually will have a minimum and start to rise again. In Fig. 4 (right) we show a sketch for three possible scenarios for $\left(\kappa \sigma^{2}\right)_{B}$ as function of $\sqrt{s_{N N}}$. A similar discussion, of course, also holds for cumulants of net electric charge fluctuations.

It thus is obvious that the observable variation of $\left(\kappa \sigma^{2}\right)_{B}$ with beam energy will crucially depend on the location of the freeze-out line relative to the critical point.

\section{Conclusions}

Higher order cumulants of net conserved charge fluctuations are sensitive to critical behavior in QCD. They provide clear-cut signatures for the existence of a critical point in the QCD phase diagram. Whether the cumulants measured experimentally can convey this information crucially depends on the relative location of the QCD transition line and the freeze-out line at which charge fluctuations are generated. In order to establish the measurement of higher order cumulants as a signature for critical behavior in QCD we need to establish that the measured fluctuations are indeed generated at a unique point in the QCD phase diagram and that they reflect thermal conditions described by equilibrium QCD. The preliminary results on net proton number and electric charge

\footnotetext{
${ }^{4}$ This feature is actually exploited when estimating the location of the critical point from ratios of subsequent expansion coefficients of the QCD partition function.
} 
fluctuations from the BES at RHIC suggest that this will in the future become possible through a systematic comparison with cumulants calculated in lattice QCD.

\section{References}

[1] M. Asakawa, K. Yazaki, Nucl. Phys. A 504, 668 (1989). A. M. Halasz et al., Phys. Rev. D 58, 096007 (1998).

[2] A. Adare et al. [PHENIX Collaboration], Phys. Rev. Lett. 104, 132301 (2010); M. Wilde [ALICE Collaboration], Nucl. Phys. A904-905 2013, 573c (2013).

[3] Y. Aoki et al., JHEP 0906, 088 (2009).

[4] A. Bazavov, et al., Phys. Rev. D 85, 054503 (2012).

[5] S. S. Adler et al. [PHENIX Collaboration], Phys. Rev. Lett. 91, 182301 (2003).

[6] C. Alt et al. [NA49 Collaboration], Phys. Rev. C 77, 024903 (2008).

[7] S. Shi [STAR Collaboration], Nucl. Phys. A904-905 2013, 895c (2013).

[8] Z. Fodor and S. D. Katz, JHEP 0404, 050 (2004).

[9] P. de Forcrand and O. Philipsen, Nucl. Phys. B 673, 170 (2003).

[10] R. V. Gavai and S. Gupta, Phys. Rev. D 78, 114503 (2008);

S. Datta, R. V. Gavai and S. Gupta, Nucl. Phys. A904-905 2013, 883c (2013).

[11] M. M. Aggarwal et al. [STAR Collaboration], Phys. Rev. Lett. 105, 022302 (2010).

[12] L. Kumar [STAR Collaboration], Nucl. Phys. A904-905 2013, 256c (2013).

[13] J. T. Mitchell [PHENIX Collaboration], Nucl. Phys. A904-905 2013, 903c (2013).

[14] P. Braun-Munzinger, J. Stachel and C. Wetterich, Phys. Lett. B 596, 61 (2004).

[15] F. Becattini, M. Bleicher, T. Kollegger, T. Schuster, J. Steinheimer and R. Stock, arXiv:1212.2431 [nucl-th]; R. Stock et al., these proceedings, arXiv:1306.4201 [nucl-th].

[16] A. Andronic et al., Nucl. Phys. A904-905 2013, 535c (2013).

[17] B. I. Abelev et al. [STAR Collaboration], Phys. Rev. C 79, 034909 (2009).

[18] O. Kaczmarek et al., Phys. Rev. D 83, 014504 (2011).

[19] G. Endrodi, Z. Fodor, S. D. Katz and K. K. Szabo, PoS LATTICE 2008, 205 (2008).

[20] F. Karsch and K. Redlich, Phys. Lett. B 695, 136 (2011).

[21] P. Braun-Munzinger, K. Redlich, J. Stachel, In *Hwa, R.C. (ed.) et al.: Quark gluon plasma* 491-599.

[22] S. Borsanyi, Z. Fodor, S. D. Katz, S. Krieg, C. Ratti and K. Szabo, JHEP 1201, 138 (2012).

[23] A. Bazavov et al. [HotQCD Collaboration], Phys. Rev. D 86, 034509 (2012).

[24] C. Ratti, R. Bellwied, M. Cristoforetti and M. Barbaro, Phys. Rev. D 85, 014004 (2012).

[25] A. Bazavov et al., arXiv:1304.7220 [hep-lat].

[26] C. Schmidt, J. Phys. Conf. Ser. 432, 012013 (2013).

[27] A. Bzdak, V. Koch and V. Skokov, Phys. Rev. C 87, 014901 (2013).

[28] S. Mukherjee and M. Wagner, these proceedings.

[29] A. Bazavov et al., Phys. Rev. Lett. 109, 192302 (2012).

[30] V. Skokov, presented at Quark Matter 2012. 